Proyecciones

Vol. 27, No 1, pp. 63-80, May 2008.

Universidad Católica del Norte

Antofagasta - Chile

\title{
DEUX EXEMPLES DE CALCUL EXPLICITE DE COHOMOLOGIE DE DOLBEAULT FEUILLETÉE
}

\author{
JIHÈNE SLIMÈNE \\ UNIVERSITÉ DE MONASTIR, TUNES
}

Received : May 2006. Accepted: May 2007

\begin{abstract}
Dans ce papier, on calcule explicitement la cohomologie de Dolbeault feuilletée pour les deux exemples de feuilletages complexes suivants : i) un feuilletage complexe linaire de dimension 1 sur le tore $\mathbf{T}^{n}$ ; ii) une fibration non localement triviale en courbes elliptiques.

In this paper, we compute explicitly the leafwise Dolbeault cohomology for two examples : i) a complex one dimensional linear foliation on the torus $\mathbf{T}^{n}$; ii) a non locally trivial fibration whose fibres are elliptic curves.
\end{abstract}

Mathematics Subject Classification : 32W05, 32G05, 32Q58, $58 A 30$

Key Words : Feuilletage complexe, $\mathcal{F}$-holomorphie, cohomologie de Dolbeault feuilletée. 


\section{Introduction}

Soit $M$ une variété différentiable munie d'un feuilletage de dimension réelle $2 m$. On dira que $\mathcal{F}$ est complexe si ses feuilles sont munies d'une structure complexe de telle sorte que les changements de coordonnées soient holomorphes (en restriction aux feuilles). On peut considérer dessus le problème du $\bar{\partial}$ le long des feuilles (ou $\bar{\partial}$ feuilleté) qui permet d'introduire la cohomologie de Dolbeault feuilletée $H_{\mathcal{F}}^{0 *}(M)$. Ce genre de questions a été abordé par exemple dans [2], [3], [4] et [6]. Dans ce travail, on se propose de calculer explicitement cette cohomologie pour les deux exemples de feuilletages complexes qui suivent.

1 - On note $\mathbf{T}^{n}=\mathbf{R}^{n} / \mathbf{Z}^{n}$ le tore de dimension $n$. Soient $X$ et $Y$ deux champs de vecteurs linéaires indépendants ; $X$ et $Y$ engendrent alors un feuilletage $\mathcal{F}$ réel orientable de dimension 2. En posant $J_{\mathcal{F}}(X)=Y$ et $J_{\mathcal{F}}(Y)=-X$, on définit sur le fibré tangent à $\mathcal{F}$ une structure presque complexe intégrable qui fait de $\mathcal{F}$ un feuilletage complexe de dimension 1. C'est un feuilletage de Lie et est défini en plus par une action libre du groupe de Lie complexe $\mathbf{C}$.

2 - On note $\mathbf{H}$ le demi-plan supérieur $\{\omega \in \mathbf{C}: \operatorname{Im} \omega>0\}$ et $\widehat{M}$ la variété complexe $\mathbf{C}^{*} \times \mathbf{H}$ qu'on munit du feuilletage holomorphe $\widehat{\mathcal{F}}$ dont les feuilles sont les facteurs $\mathbf{C}^{*} \times\{\omega\}$ avec $\omega$ variant dans $\mathbf{H}$. Ce feuilletage est invariant par le biholomorphisme $\gamma:(z, \omega) \in \mathbf{C}^{*} \times \mathbf{H} \longmapsto\left(e^{-i \varphi(\omega)} z, \omega\right) \in$ $\mathbf{C}^{*} \times \mathbf{H}$ où $\varphi$ est un biholomorphisme de $\mathbf{H}$. Il induit donc un feuilletage holomorphe (a fortiori complexe) $\mathcal{F}$ de dimension 1 sur la variété quotient $M=\widehat{M} / \Gamma$ où $\Gamma$ est le groupe des automorphismes de $(\widehat{M}, \widehat{\mathcal{F}})$ engendré par $\gamma$. La variété $M$ et le feuilletage $\mathcal{F}$ sont différentiablement le produit par $\mathbf{H}$ d'une courbe elliptique $\Sigma$. Mais du point de vue complexe $\mathcal{F}$ est loin d'être un produit : deux feuilles $\Sigma_{\omega}$ et $\Sigma_{\zeta}$ sont holomorphiquement équivalentes si, et seulement si, il existe une matrice $B \in \mathrm{SL}(2, \mathbf{Z})$ telle que $\zeta=B \omega$. Ce feuilletage a des courbes elliptiques comme feuilles qui épuisent toutes les structures complexes possibles.

Sauf mention expresse du contraire ou précision, tous les objets considérés (variétés, applications, feuilletages...) sont supposés être de classe $C^{\infty}$.

\section{Feuilletages complexes}

Soit $M$ une variété différentiable munie d'un feuilletage $\mathcal{F}$ de dimension réelle $2 m$. On notera $T \mathcal{F}$ le fibré tangent à $\mathcal{F}$. 
Définition 2.1. On dira que $\mathcal{F}$ est complexe s'il peut être défini par un recouvrement ouvert $\left\{U_{i}\right\}$ de $M$ et des difféomorphismes $\phi_{i}: \Omega_{i} \times \mathcal{O}_{i} \longrightarrow U_{i}$ (où $\Omega_{i}$ est un ouvert de $\mathbf{C}^{\mathbf{m}}$ et $\mathcal{O}_{i}$ un ouvert de $\mathbf{R}^{\mathbf{n}}$ ) tels que les changements de coordonnées :

$$
\phi_{i j}=\phi_{j}^{-1} \circ \phi_{i}:(z, t) \in \phi_{i}^{-1}\left(U_{i} \cap U_{j}\right) \longrightarrow\left(z^{\prime}, t^{\prime}\right) \in \phi_{j}^{-1}\left(U_{i} \cap U_{j}\right)
$$

soient de la forme $\left(z^{\prime}, t^{\prime}\right)=\left(\phi_{i j}^{1}(z, t), \phi_{i j}^{2}(t)\right)$ avec $\phi_{i j}^{1}$ holomorphe en z pour $t$ fixé.

Soient $(M, \mathcal{F})$ et $\left(M^{\prime}, \mathcal{F}^{\prime}\right)$ deux feuilletages complexes. On appelle morphisme de $(M, \mathcal{F})$ vers $\left(M^{\prime}, \mathcal{F}^{\prime}\right)$ toute application $f: M \longrightarrow M^{\prime}$ de classe $C^{\infty}$ et telle que l'image de toute feuille $F$ de $\mathcal{F}$ est contenue dans une feuille $F^{\prime}$ de $\mathcal{F}^{\prime}$ et l'application $f: F \longrightarrow F^{\prime}$ est holomorphe. On dira qu'un morphisme $f:(M, \mathcal{F}) \longrightarrow\left(M^{\prime}, \mathcal{F}^{\prime}\right)$ est un isomorphisme de feuilletages complexes si c'est un difféomorphisme qui est un biholomorphisme sur les feuilles. Lorsque $M=M^{\prime}$ et $\mathcal{F}=\mathcal{F}^{\prime}$ on parlera simplement d'automorphisme de $(M, \mathcal{F})$. On notera $\operatorname{Aut}(M, \mathcal{F})$ l'ensemble des automorphismes de $(M, \mathcal{F})$ qui est un groupe pour la composition des applications.

\subsection{Cohomologie de Dolbeault feuilletée}

Une structure complexe sur les feuilles définit bien sûr une structure presque complexe sur $\mathcal{F}$ i.e. un endomorphisme $J_{\mathcal{F}}: T \mathcal{F} \longrightarrow T \mathcal{F}$ tel que $J_{\mathcal{F}}^{2}=$ $-\mathrm{id}_{T \mathcal{F}}$. Considérons le complexifié $T \mathcal{F} \otimes \mathbf{C}$ du fibré $T \mathcal{F}$ et notons respectivement $T^{10} \mathcal{F}$ et $T^{01} \mathcal{F}$ les sous-fibrés propres associés respectivement aux valeurs propres $i$ et $-i$ de l'automorphisme $J_{\mathcal{F}}$. On a une décomposition en somme directe $T \mathcal{F} \otimes \mathbf{C}=T^{10} \mathcal{F} \oplus T^{01} \mathcal{F}$. Pour tout $r \in \mathbf{N}$, on note $\Omega_{\mathcal{F}}^{r}(M)$ l'espace des sections $C^{\infty}$ du fibré $\Lambda^{r} T \mathcal{F}^{*} \otimes \mathbf{C}$; en fixant un complémentaire du fibré $T \mathcal{F}$ dans $T M$, on peut voir les éléments de $\Omega_{\mathcal{F}}^{r}(M)$ comme des formes différentielles feuilletées sur $(M, \mathcal{F})$ (à coefficients complexes). Pour tout $r \in\{0, \cdots, 2 m\}$, on a une décomposition en somme directe :

$$
\Omega_{\mathcal{F}}^{r}(M)=\bigoplus_{p+q=r} \Omega_{\mathcal{F}}^{p, q}(M)
$$

Un élément $\alpha \in \Omega_{\mathcal{F}}^{p, q}(M)$ est appelé forme feuilletée de type $(p, q)$. La différentielle extérieure le long des feuilles $d_{\mathcal{F}}$ se décompose en une somme d'opérateurs $d_{\mathcal{F}}=\partial_{\mathcal{F}}+\bar{\partial}_{\mathcal{F}}$ respectivement de types $(1,0)$ et $(0,1)$. 
L'opérateur :

$$
\bar{\partial}_{\mathcal{F}}: \alpha \in \Omega_{\mathcal{F}}^{p, q}(M) \longmapsto \bar{\partial}_{\mathcal{F}} \alpha \in \Omega_{\mathcal{F}}^{p, q+1}(M)
$$

est dit opérateur de Cauchy-Riemann le long des feuilles de $(M, \mathcal{F})$. C'est un opérateur elliptique le long des feuilles mais il n'est jamais elliptique sur toute la variété au sens classique sauf si $\operatorname{dim}_{\mathbf{R}} \mathcal{F}=\operatorname{dim} M$. Il vérifie $\bar{\partial}_{\mathcal{F}} \circ \bar{\partial}_{\mathcal{F}}=0$; pour tout $p$ fixé dans $\{0, \ldots, m\}$, on obtient donc un complexe différentiel :

$$
0 \longrightarrow \Omega_{\mathcal{F}}^{p, 0}(M) \stackrel{\bar{\partial}_{\mathcal{F}}}{\longrightarrow} \Omega_{\mathcal{F}}^{p, 1}(M) \stackrel{\bar{\partial}_{\mathcal{F}}}{\longrightarrow} \cdots \stackrel{\bar{\partial}_{\mathcal{F}}}{\longrightarrow} \Omega_{\mathcal{F}}^{p, m-1}(M) \stackrel{\bar{\partial}_{\mathcal{F}}}{\longrightarrow} \Omega_{\mathcal{F}}^{p, m}(M) \longrightarrow 0
$$

appelé complexe de Dolbeault feuilleté (ou complexe du $\bar{\partial}_{\mathcal{F}}$ ) de $\mathcal{F}$. On pose :

$$
Z_{\mathcal{F}}^{p, q}(M)=\operatorname{noyau}\left\{\Omega_{\mathcal{F}}^{p, q}(M) \stackrel{\bar{\partial}_{\mathcal{F}}}{\longrightarrow} \Omega_{\mathcal{F}}^{p, q+1}(M)\right\}
$$

et

$$
B_{\mathcal{F}}^{p, q}(M)=\text { image }\left\{\Omega_{\mathcal{F}}^{p, q-1}(M) \stackrel{\bar{\partial}_{\mathcal{F}}}{\longrightarrow} \Omega_{\mathcal{F}}^{p, q}(M)\right\} .
$$

La cohomologie de ce complexe $H_{\mathcal{F}}^{p, *}(M)=Z_{\mathcal{F}}^{p, *}(M) / B_{\mathcal{F}}^{p, *}(M)$ est appelée $\bar{\partial}_{\mathcal{F}}$-cohomologie ou cohomologie de Dolbeault feuilletée.

L'espace vectoriel topologique $H_{\mathcal{F}}^{p, q}(M)$ (les espaces de formes feuilletées de type $(p, q)$ sont munis de la topologie $\left.C^{\infty}\right)$ peut ne pas être séparé ! On appelle alors cohomologie de Dolbeault feuilletée réduite le quotient $\bar{H}_{\mathcal{F}}^{p, q}(M)=Z_{\mathcal{F}}^{p, q}(M) / \overline{B_{\mathcal{F}}^{p, q}(M)}$ où $\overline{B_{\mathcal{F}}^{p, q}(M)}$ est l'adhérence de $B_{\mathcal{F}}^{p, q}(M)$.

Problme du $\bar{\partial}_{\mathcal{F}}$ 2.1. Étant donnée une forme feuilletée $\beta \in Z_{\mathcal{F}}^{p, q}(M)$, existe-t-il une forme feuilletée $\alpha \in \Omega_{\mathcal{F}}^{p, q-1}(M)$ telle que $\bar{\partial}_{\mathcal{F}} \alpha=\beta$ ?

La cohomologie de Dolbeault feuilletée mesure donc l'obstruction à l'existence des solutions pour le problème du $\bar{\partial}_{\mathcal{F}}$. Localement, ce problème admet toujours une solution ; plus précisément, on a une version feuilletée du Lemme de Dolbeault-Grothendieck qui est l'équivalent du lemme de Poincaré pour la cohomologie de de Rham. La démonstration consiste à adapter au cas paramétré celle du cas classique bien connue.

Lemme 2.2. (Dolbeault-Grothendieck feuilleté) Tout point de $M$ admet un voisinage ouvert $U$ distingué pour $\mathcal{F}$ tel que, pour tout $p=0, \ldots, m$, on ait $H_{\mathcal{F}}^{p, q}(U)=0$ pour $q \geq 1$.

On peut aussi décrire $H_{\mathcal{F}}^{p, *}(M)$ à l'aide d'un faisceau qui joue un rôle analogue au faisceau des germes de formes holomorphes sur une variété analytique complexe. 
Définition 2.3. Une $p$-forme $\alpha$ est dite $\mathcal{F}$ - holomorphe, si elle est feuilletée de type $(p, 0)$ et vérifie $\bar{\partial}_{\mathcal{F}} \alpha=0$.

Localement, une $p$-forme $\mathcal{F}$-holomorphe s'écrit $\alpha=\sum \alpha_{j_{1} \cdots j_{p}}(z, t) d z_{j_{1}} \wedge$ $\cdots \wedge d z_{j_{p}}$ avec $\alpha_{j_{1} \cdots j_{p}}$ holomorphe en $z$.

Soient $\mathcal{H}_{\mathcal{F}}^{p}$ le faisceau des germes de $p$-formes $\mathcal{F}$-holomorphes sur $M$ et $\widetilde{\Omega}_{\mathcal{F}}^{p, q}$ celui des germes de formes différentielles de type $(p, q)$ sur $\mathcal{F}$; ce dernier est un faisceau fin sur $M$.

Proposition 2.4. La suite $0 \longrightarrow \mathcal{H}_{\mathcal{F}}^{p} \hookrightarrow \widetilde{\Omega}_{\mathcal{F}}^{p, 0} \stackrel{\bar{\partial}_{\mathcal{F}}}{\longrightarrow} \cdots \stackrel{\bar{\partial}_{\mathcal{F}}}{\longrightarrow} \widetilde{\Omega}_{\mathcal{F}}^{p, m} \longrightarrow 0$ est une résolution fine de $\mathcal{H}_{\mathcal{F}}^{p}$. On a alors $H^{q}\left(M, \mathcal{H}_{\mathcal{F}}^{p}\right)=H_{\mathcal{F}}^{p, q}(M)$, pour tous $p, q=0,1, \ldots m$.

Si $n \geq 1$, cette résolution n'est pas elliptique ; elle l'est seulement le long des feuilles. La cohomologie $H^{*}\left(M, \mathcal{H}_{\mathcal{F}}^{p}\right)$ n'est donc pas toujours de dimension finie même si $M$ est compacte.

Pour $p=0$, on notera $\mathcal{H}_{\mathcal{F}}$ le faisceau $\mathcal{H}_{\mathcal{F}}^{0}$; ses sections sont les fonctions $C^{\infty}$ qui sont $\mathcal{F}$-holomorphes. Pour tout ouvert $U \subset M$, l'espace $H^{0}\left(U, \mathcal{H}_{\mathcal{F}}\right)$ est constitué par les fonctions $C^{\infty}$ et $\mathcal{F}$-holomorphes sur $U$. On le notera $\mathcal{H}_{\mathcal{F}}(U)$ et simplement $\mathcal{H}(U)$ si $\mathcal{F}$ est de codimension 0 i.e. $M$ est complexe et est la seule feuille de $\mathcal{F}$.

\subsection{Une méthode de calcul}

On se donne un feuilletage complexe $\widetilde{\mathcal{F}}$ de dimension $m$ sur une variété $\widetilde{M}$ et $\Gamma$ un groupe dénombrable opérant librement et proprement sur $\widetilde{M}$ par automorphismes de $\widetilde{\mathcal{F}}$ (en tant que feuilletage complexe). Alors la variété quotient $M=\widetilde{M} / \Gamma$ est munie du feuilletage induit $\mathcal{F}$ qui est complexe de dimension $m$. Notons $\pi: \widetilde{M} \longrightarrow M$ la projection de revêtement ; c'est un morphisme de $(\widetilde{M}, \widetilde{\mathcal{F}})$ sur $(M, \mathcal{F})$. L'image réciproque $\pi^{*}\left(\mathcal{H}_{\mathcal{F}}\right)$ du faisceau $\mathcal{H}_{\mathcal{F}}$ n'est rien d'autre que le faisceau $\mathcal{H}_{\widetilde{\mathcal{F}}}$. Il existe alors une suite spectrale $E_{r}$ dont le terme $E_{2}$ est donné par :

$$
E_{2}^{k \ell}=H^{k}\left(\Gamma, H^{\ell}\left(\widetilde{M}, \mathcal{H}_{\widetilde{\mathcal{F}}}\right)\right)
$$

et convergeant vers $H^{*}\left(M, \mathcal{H}_{\mathcal{F}}\right)$. Les espaces vectoriels $H^{\ell}\left(\widetilde{M}, \mathcal{H}_{\widetilde{\mathcal{F}}}\right)$ sont vus comme des $\Gamma$-modules via l'action induite sur $H^{\ell}\left(\widetilde{M}, \mathcal{H}_{\widetilde{\mathcal{F}}}\right)$ par celle de $\Gamma \operatorname{sur} \mathcal{H}_{\widetilde{\mathcal{F}}}(\widetilde{M}):$

$$
(\gamma, f) \in \Gamma \times \mathcal{H}_{\widetilde{\mathcal{F}}}(\widetilde{M}) \longmapsto f \circ \gamma^{-1} \in \mathcal{H}_{\widetilde{\mathcal{F}}}(\widetilde{M}) .
$$


(Cette suite spectrale résulte de la théorie des foncteurs dérivés de Grothendieck [7]. On peut en trouver un exposé dans [1].) Si $\widetilde{M}$ est acyclique i.e. :

$$
H^{l}\left(\widetilde{\mathcal{M}}, \mathcal{H}_{\widetilde{F}}\right)=\left\{\begin{array}{lll}
\mathcal{H}_{\widetilde{F}} & \text { si } & l=0 \\
0 & \text { si } & l \geq 1
\end{array}\right.
$$

la suite $E_{r}$ converge au terme $E_{2}$ et on a :

$$
H^{k}\left(M, \mathcal{H}_{\mathcal{F}}\right)=H^{k}\left(\Gamma, \mathcal{H}_{\widetilde{\mathcal{F}}}(\widetilde{M})\right)
$$

Lorsque le groupe $\Gamma$ est isomorphe à $\mathbf{Z}$ et est engendré par un élément $\gamma$, tous les groupes de cohomologie $H^{k}\left(\Gamma, \mathcal{H}_{\widetilde{\mathcal{F}}}\right)$ sont nuls pour $k \geq 2$ et $H^{1}\left(\Gamma, \mathcal{H}_{\widetilde{\mathcal{F}}}\right)=\mathcal{H}_{\widetilde{\mathcal{F}}}(\widetilde{M}) / \mathcal{C}$ où $\mathcal{C}$ est le sous-espace de $\mathcal{H}_{\widetilde{\mathcal{F}}}(\widetilde{M})$ engendré par les éléments de la forme $h-h \circ \gamma$ avec $h \in \mathcal{H}_{\widetilde{\mathcal{F}}}(\widetilde{M})$. Ce qui nous amènera souvent à résoudre l'équation cohomologique discrète $f-f \circ \gamma=g$ dans l'espace $\mathcal{H}_{\widetilde{\mathcal{F}}}(\widetilde{M})$.

Tout le reste de ce papier sera consacré au calcul (souvent explicite) du premier groupe de cohomologie de Dolbeault feuilletée pour les exemples de feuilletages complexes mentionnés dans l'introduction.

\section{Feuilletage complexe linéaire sur le tore $\mathbf{T}^{n}$}

Soit $n \geq 3$ un entier ; munissons l'espace vectoriel $\mathbf{R}^{n}$ de son produit scalaire habituel noté $\langle$,$\rangle et de la norme associée |\cdot|$. Le tore $\mathbf{T}^{n}$ est obtenu comme le quotient de $\mathbf{R}^{n}$ par son réseau standard $\mathbf{Z}^{n}$. Pour $\mathbf{m} \in \mathbf{Z}^{n}$, on note $e_{\mathbf{m}}$ la fonction $e_{\mathbf{m}}(x)=e^{2 i \pi\langle\mathbf{m}, x\rangle}$. Une fonction sur $\mathbf{T}^{n}$ n'est rien d'autre qu'une fonction $f: \mathbf{R}^{n} \longrightarrow \mathbf{C}$ qui vérifie $f(x+\mathbf{m})=f(x)$ pour tous $x \in \mathbf{R}^{n}$ et $\mathbf{m} \in \mathbf{Z}^{n}$. Si $f$ est intégrable, elle peut être développée en série de Fourier :

$$
\sum_{\mathbf{m} \in \mathbf{Z}^{n}} f_{\mathbf{m}} e_{\mathbf{m}}(x)
$$

où les $f_{\mathbf{m}}$ sont ses coefficients de Fourier donnés par les formules intégrales:

$$
f_{\mathbf{m}}=\int_{\mathbf{T}^{n}} f(x) e^{-2 i \pi\langle\mathbf{m}, x\rangle} d x .
$$

Si en plus $f$ est de carré intégrable, les coefficients $f_{\mathbf{m}}$ vérifient la condition de convergence $\sum_{\mathbf{m} \in \mathbf{Z}^{n}}\left|f_{\mathbf{m}}\right|^{2}<+\infty$. 
Pour tout $r \in \mathbf{N}$, on note $W^{1, r}$ l'espace des fonctions $f$ sur le tore $\mathbf{T}^{n}$ données par leurs coefficients de Fourier $\left(f_{\mathbf{m}}\right)_{\mathbf{m} \in \mathbf{Z}^{n}}$ vérifiant la condition $\sum_{\mathbf{m} \in \mathbf{Z}^{n} \backslash\{\mathbf{0}\}}|\mathbf{m}|^{2 r}\left|f_{\mathbf{m}}\right|^{2}<+\infty$. De même, $W^{2, r}$ sera l'espace des fonctions $f$ sur le tore $\mathbf{T}^{n}$ données par leurs coefficients de Fourier $\left(f_{\mathbf{m}}\right)_{\mathbf{m} \in \mathbf{Z}^{n}}$ vérifiant la condition $\sum_{\mathbf{m} \in \mathbf{Z}^{n} \backslash\{\mathbf{0}\}}|\mathbf{m}|^{2 r}\left|f_{\mathbf{m}}\right|^{2}<+\infty$. Ce sont des espaces complets pour les normes :

$$
\|f\|_{1, r}=\left|f_{\mathbf{0}}\right|+\sum_{\mathbf{m} \in \mathbf{Z}^{n} \backslash\{\mathbf{0}\}}|\mathbf{m}|^{r}\left|f_{\mathbf{m}}\right| \quad \text { pour } f \in W^{1, r}
$$

et

$$
\|f\|_{2, r}=\sqrt{\left|f_{\mathbf{0}}\right|^{2}+\sum_{\mathbf{m} \in \mathbf{Z}^{n} \backslash\{\mathbf{0}\}}|\mathbf{m}|^{2 r}\left|f_{\mathbf{m}}\right|^{2}} \quad \text { pour } f \in W^{2, r}
$$

L'espace $W^{2, r}$ est le $r^{\text {ème }}$ espace de Sobolev du tore $\mathbf{T}^{n}$; il a une structure d'espace de Hilbert donnée par le produit hermitien :

$$
\langle f, g\rangle_{r}=f_{\mathbf{0}} \bar{g}_{\mathbf{0}}+\sum_{\mathbf{m} \in \mathbf{Z}^{n} \backslash\{\mathbf{0}\}}|\mathbf{m}|^{2 r} f_{\mathbf{m}} \bar{g}_{\mathbf{m}}
$$

On a des inclusions naturelles :

$$
C^{\infty}\left(\mathbf{T}^{n}\right) \subset \cdots \subset W^{1, r+1} \subset W^{1, r} \subset \cdots \subset W^{1,0}
$$

et

$$
C^{\infty}\left(\mathbf{T}^{n}\right) \subset \cdots \subset W^{2, r+1} \subset W^{2, r} \subset \cdots \subset W^{2,0}=L^{2}\left(\mathbf{T}^{n}\right) .
$$

La proposition suivante est facile à démontrer.

Proposition 3.1. Soit $f=\sum_{\mathbf{m} \in \mathbf{Z}^{n}} f_{\mathbf{m}} e_{\mathbf{m}}$ une série (les $f_{\mathbf{m}}$ sont des nombres complexes). Alors les assertions i), ii) et iii) qui suivent sont équivalentes :

i) $f$ est une fonction de classe $C^{\infty}$;

ii) pour tout $r \in \mathbf{N}^{*}$, la série $\sum_{\mathbf{m} \in \mathbf{Z}^{n}}|\mathbf{m}|^{2 r}\left|f_{\mathbf{m}}\right|^{2}$ est convergente ;

iii) pour tout $r \in \mathbf{N}^{*}$, la série $\sum_{\mathbf{m} \in \mathbf{Z}^{n}}|\mathbf{m}|^{r}\left|f_{\mathbf{m}}\right|$ est convergente.

Pour tout $r \in \mathbf{N}$, les injections $j_{1, r}: W^{1, r+1} \hookrightarrow W^{1, r}$ et $j_{2, r}: W^{2, r+1} \hookrightarrow$ $W^{2, r}$ sont des opérateurs compacts. 
Les trois premiers points de cette proposition disent :

$\bigcap_{r \in \mathbf{N}} W^{1, r}=\bigcap_{r \in \mathbf{N}} W^{2, r}=C^{\infty}\left(\mathbf{T}^{n}\right)$

Soient $X=\sum_{i=1}^{n} \kappa_{i} \frac{\partial}{\partial x_{i}}$ et $Y=\sum_{j=1}^{n} \nu_{j} \frac{\partial}{\partial x_{j}}$ deux champs de vecteurs linéaires et indépendants sur $\mathbf{T}^{n}$. Ils engendrent un sous-fibré intégrable du fibré tangent à $\mathbf{T}^{n}$ et définissent ainsi un feuilletage $\mathcal{F}$ sur $\mathbf{T}^{n}$ de dimension réelle 2. Considérons la structure presque complexe le long des feuilles définie par $J_{\mathcal{F}}(X)=Y$ et $J_{\mathcal{F}}(Y)=-X ; \mathcal{F}$ étant un feuilletage orientable par surfaces, $J_{\mathcal{F}}$ est intégrable et confère à $\mathcal{F}$ une structure complexe.

Les fibrés $T^{10} \mathcal{F}$ et $T^{01} \mathcal{F}$ sont engendrés respectivement par les champs:

$$
Z=\frac{1}{2}(X-i Y)=\frac{1}{2}\left\{\left(\kappa_{1} \frac{\partial}{\partial x_{1}}+\cdots+\kappa_{n} \frac{\partial}{\partial x_{n}}\right)-i\left(\nu_{1} \frac{\partial}{\partial x_{1}}+\cdots+\nu_{n} \frac{\partial}{\partial x_{n}}\right)\right\}
$$

et

$\bar{Z}=\frac{1}{2}(X+i Y)=\frac{1}{2}\left\{\left(\kappa_{1} \frac{\partial}{\partial x_{1}}+\cdots+\kappa_{n} \frac{\partial}{\partial x_{n}}\right)+i\left(\nu_{1} \frac{\partial}{\partial x_{1}}+\cdots+\nu_{n} \frac{\partial}{\partial x_{n}}\right)\right\}$.

Ils forment une base $(Z, \bar{Z})$ du complexifié $T \mathcal{F} \otimes \mathbf{C}$ du fibré tangent au feuilletage. Soient $\kappa^{\prime}=\left(\kappa_{1}^{\prime}, \cdots, \kappa_{n}^{\prime}\right)$ et $\nu^{\prime}=\left(\nu_{1}^{\prime}, \cdots, \nu_{n}^{\prime}\right)$ des vecteurs de $\mathbf{R}^{n}$ tels que :

$$
\left\langle\kappa, \kappa^{\prime}\right\rangle=1 \quad\left\langle\nu, \kappa^{\prime}\right\rangle=0 \quad\left\langle\kappa, \nu^{\prime}\right\rangle=0 \quad \text { et } \quad\left\langle\nu, \nu^{\prime}\right\rangle=1 .
$$

Alors $(Z, \bar{Z})$ admet pour base duale $(\omega, \bar{\omega})$ où $\omega$ et $\bar{\omega}$ sont les 1-formes feuilletées de types respectivement $(1,0)$ et $(0,1)$ et données explicitement par :

$$
\omega=\left(\kappa_{1}^{\prime} d x_{1}+\cdots+\kappa_{n}^{\prime} d x_{n}\right)+i\left(\nu_{1}^{\prime} d x_{1}+\cdots+\nu_{n}^{\prime} d x_{n}\right)
$$

et

$$
\bar{\omega}=\left(\kappa_{1}^{\prime} d x_{1}+\cdots+\kappa_{n}^{\prime} d x_{n}\right)-i\left(\nu_{1}^{\prime} d x_{1}+\cdots+\nu_{n}^{\prime} d x_{n}\right) .
$$

Le complexe de Dolbeault feuilleté s'écrit :

$$
0 \longrightarrow \Omega_{\mathcal{F}}^{0,0}\left(\mathbf{T}^{n}\right) \stackrel{\bar{\partial}_{\mathcal{F}}}{\longrightarrow} \Omega_{\mathcal{F}}^{0,1}\left(\mathbf{T}^{n}\right) \longrightarrow 0
$$

où l'opérateur $\bar{\partial}_{\mathcal{F}}$ est donné par : 


$$
\bar{\partial}_{\mathcal{F}} f=\frac{1}{2}\left\{\left(\kappa_{1} \frac{\partial f}{\partial x_{1}}+\cdots+\kappa_{n} \frac{\partial f}{\partial x_{n}}\right)+i\left(\nu_{1} \frac{\partial f}{\partial x_{1}}+\cdots+\nu_{n} \frac{\partial f}{\partial x_{n}}\right)\right\} \otimes \bar{\omega} .
$$

avec

$$
\Omega_{\mathcal{F}}^{0, *}\left(\mathbf{T}^{n}\right)= \begin{cases}C^{\infty}\left(\mathbf{T}^{n}\right) & \text { si* }=0 \\ C^{\infty}\left(\mathbf{T}^{n}\right) \otimes \bar{\omega} & \text { si* }=1\end{cases}
$$

On suppose que les $\kappa_{i}$ (ainsi que les $\nu_{i}$ ) sont $Q$-indépendants. Le feuilletage $\mathcal{F}$ est alors à feuilles denses.

Définitions 3.2. Soient $\nu, \kappa \in \mathbf{R}^{n}$ deux vecteurs. On dira que :

- le vecteur $\nu$ est diophantien s'il existe $\delta>0$ et $\tau>0$ tels que :

$$
|\langle\nu, \mathbf{m}\rangle| \geq \frac{\delta}{|\mathbf{m}|^{\tau}} \text { pour tout } \mathbf{m} \in \mathbf{Z}^{n} \backslash\{0\} ;
$$

- le couple $(\nu, \kappa)$ est de Liouville s'il existe $\delta$ tel que, pour toute suite strictement croissante $\left(\tau_{k}\right)_{k}$ dans $\mathbf{N}^{*}$, il existe une suite infinie $\left(\mathbf{m}_{k}\right)_{k}$ dans $\mathbf{Z}^{n} \backslash\{\mathbf{0}\}$ vérifiant :

$$
\left|\left\langle\nu, \mathbf{m}_{k}\right\rangle+i\left\langle\kappa, \mathbf{m}_{k}\right\rangle\right| \leq \frac{\delta}{\left|\mathbf{m}_{k}\right|^{\tau_{k}}}
$$

Un champ de vecteurs $X=\sum_{j=1}^{n} \nu_{j} \frac{\partial}{\partial x_{j}}$ est dit diophantien si $\nu=\left(\nu_{1}, \ldots, \nu_{n}\right)$ l'est.

Si $X$ ou $Y$ est diophantien, on dira que $\mathcal{F}$ est un feuilletage diophantien. Si le couple de champs $(X, Y)$ avec $X=\sum_{j=1}^{n} \nu_{j} \frac{\partial}{\partial x_{j}}$ et $Y=\sum_{j=1}^{n} \kappa_{j} \frac{\partial}{\partial x_{j}}$ définissant $\mathcal{F}$ est tel que le couple $(\nu, \kappa)$ est de Liouville, on dira que $\mathcal{F}$ est un feuilletage de Liouville.

On définit une forme linéaire continue $\mathcal{L}: C^{\infty}\left(\mathbf{T}^{n}\right) \longrightarrow \mathbf{C}$ par $\mathcal{L}(g)=g_{\mathbf{0}}$ pour toute fonction $g=\sum_{\mathbf{m} \in \mathbf{Z}^{n}} g_{\mathbf{m}} e_{\mathbf{m}}$. On peut aussi interpréter $\mathcal{L}$ comme un opérateur sur $C^{\infty}\left(\mathbf{T}^{n}\right)$ qui à $g$ associe la fonction $\mathcal{L}(g) \mathbf{1}$ où $\mathbf{1}$ est la fonction constante égale à 1 ; c'est donc un opérateur compact car de rang fini (son rang est 1). Son noyau $\mathcal{N}$ est fermé et tel que $C^{\infty}\left(\mathbf{T}^{n}\right)=\mathcal{N} \oplus \mathbf{C} \cdot \mathbf{1}$. Notons $P$ la première projection $C^{\infty}\left(\mathbf{T}^{n}\right)=\mathcal{N} \oplus \mathbf{C} \cdot \mathbf{1} \longrightarrow \mathcal{N}$. Elle vérifie $P \oplus \mathcal{L}=I$ (où $I$ est l'identité de $\left.C^{\infty}\left(\mathbf{T}^{n}\right)\right)$. On a alors le : 
Théorème 3.3. Soit $\mathcal{F}$ le feuilletage linéaire sur le tore $\mathbf{T}^{n}$ défini comme précédemment.

i) Supposons que le feuilletage $\mathcal{F}$ est diophantien. Alors il existe un opérateur borné

$G: C^{\infty}\left(\mathbf{T}^{n}\right) \longrightarrow C^{\infty}\left(\mathbf{T}^{n}\right)$ tel que $G \bar{\partial}_{\mathcal{F}}=I-\mathcal{L}$. Il en découle que :

$$
H_{\mathcal{F}}^{0, q}\left(\mathbf{T}^{n}\right)= \begin{cases}\mathbf{C} & \text { si } q=0 \\ \mathbf{C} \otimes \bar{\omega} & \text { si } q=1 \\ 0 & \text { si } q \geq 2\end{cases}
$$

ii) Supposons $\mathcal{F}$ de Liouville. Alors on a toujours

$H_{\mathcal{F}}^{0,0}\left(\mathbf{T}^{n}\right)=\mathbf{C}, H_{\mathcal{F}}^{0, q}\left(\mathbf{T}^{n}\right)=0$ pour $q \geq 2$ et l'espace vectoriel topologique $H_{\mathcal{F}}^{0,1}\left(\mathbf{T}^{n}\right)$ est de dimension infinie et est non séparé. Mais $\bar{H}_{\mathcal{F}}^{0,1}\left(\mathbf{T}^{n}\right)$ est isomorphe à $\mathbf{C}$ et est engendré par $\bar{\omega}$.

Proof. Quelle que soit la nature arithmétique de $\mathcal{F}$ (diophantien ou de Liouville), on a $H_{\mathcal{F}}^{0,0}\left(\mathbf{T}^{n}\right)=\mathbf{C}$ car les feuilles sont denses et $H_{\mathcal{F}}^{0, q}\left(\mathbf{T}^{n}\right)=0$ pour $q \geq 2$ car $\operatorname{dim}_{\mathbf{C}} \mathcal{F}=1$. Il n'y a en fait que le cas $q=1$ à examiner.

Déterminons l'espace $H_{\mathcal{F}}^{0,1}\left(\mathbf{T}^{n}\right)$ en cherchant une condition nécessaire et suffisante sur $g \in C^{\infty}\left(\mathbf{T}^{n}\right), g=\sum_{\mathbf{m} \in \mathbf{Z}^{n}} g_{\mathbf{m}} e_{\mathbf{m}}$ pour qu'il existe une fonction $f \in C^{\infty}\left(\mathbf{T}^{n}\right), f=\sum_{\mathbf{m} \in \mathbf{Z}^{n}} f_{\mathbf{m}} e_{\mathbf{m}}$ vérifiant l'équation aux dérivées partielles :

$$
\frac{1}{2}\left\{\left(\nu_{1} \frac{\partial f}{\partial x_{1}}+\cdots+\nu_{n} \frac{\partial f}{\partial x_{n}}\right)+i\left(\kappa_{1} \frac{\partial f}{\partial x_{1}}+\cdots+\kappa_{n} \frac{\partial f}{\partial x_{n}}\right)\right\}=g
$$

En identifiant les coefficients de Fourier des deux membres de l'égalité (3) on aura, pour tout $\mathbf{m} \in \mathbf{Z}^{n}$ :

$$
\left(E_{\mathbf{m}}\right) \quad i \pi(\langle\nu, \mathbf{m}\rangle+i\langle\kappa, \mathbf{m}\rangle) f_{\mathbf{m}}=g_{\mathbf{m}}
$$

Pour $\mathbf{m}=\mathbf{0}$, le premier membre de l'équation $\left(E_{\mathbf{m}}\right)$ est nul. Une condition nécessaire d'existence d'une solution est donc $g_{\mathbf{0}}=0$. On pose alors :

$$
f_{\mathbf{m}}= \begin{cases}0 & \text { si } \mathbf{m}=\mathbf{0} \\ \frac{-i g \mathbf{m}}{\pi(\langle\nu, \mathbf{m}\rangle+i\langle\kappa, \mathbf{m}\rangle)} & \text { si } \mathbf{m} \in \mathbf{Z}^{n} \backslash\{\mathbf{0}\}\end{cases}
$$


Reste à montrer que la famille des $f_{\mathbf{m}}$ définit effectivement une fonction $f \in C^{\infty}\left(\mathbf{T}^{n}\right)$. Cela va dépendre de la "nature arithmétique" du feuilletage $\mathcal{F}$.

\section{i) $\underline{\mathcal{F} \text { diophantien }}$}

Pour $\mathbf{m} \in \mathbf{Z}^{n} \backslash\{\mathbf{0}\}$, on a $\left|f_{\mathbf{m}}\right| \leq \frac{|\langle\nu, \mathbf{m}\rangle|+|\langle\kappa, \mathbf{m}\rangle|}{\pi\left(\langle\nu, \mathbf{m}\rangle^{2}+\langle\kappa, \mathbf{m}\rangle^{2}\right)}\left|g_{\mathbf{m}}\right|$. L'inégalité de Cauchy-Schwarz donne :

$$
\left|f_{\mathbf{m}}\right| \leq|\mathbf{m}| \frac{C}{\left(\langle\nu, \mathbf{m}\rangle^{2}+\langle\kappa, \mathbf{m}\rangle^{2}\right)}\left|g_{\mathbf{m}}\right|
$$

avec $C=\frac{|\nu|+|\kappa|}{\pi}$. Ainsi $\left|f_{\mathbf{m}}\right| \leq \frac{C}{\langle\vartheta, \mathbf{m}\rangle^{2}}|\mathbf{m}| \cdot\left|g_{\mathbf{m}}\right|$ avec $\vartheta \in\{\kappa, \nu\} \quad(\vartheta$ est celui des deux vecteurs $\nu$ ou $\kappa$ qui est diophantien). Comme $\vartheta$ est diophantien, il existe $\delta>0$ et $\tau>0$ tels que $|\langle\vartheta, \mathbf{m}\rangle| \geq \frac{\delta}{|\mathbf{m}|^{\tau}}$, pour tout $\mathbf{m} \in \mathbf{Z}^{n} \backslash\{0\}$. On a donc, pour tout $s \in \mathbf{N}$ :

$$
|\mathbf{m}|^{s}\left|f_{\mathbf{m}}\right| \leq \beta|\mathbf{m}|^{s+1+2 \tau}\left|g_{\mathbf{m}}\right|
$$

avec $\beta=\frac{C}{\delta^{2}}$. La série $\sum_{\mathbf{m} \in \mathbf{Z}^{n}}|\mathbf{m}|^{s}\left|f_{\mathbf{m}}\right|$ converge donc, c'est-à-dire que la fonction $f$ est de classe $C^{\infty}$ sur $\mathbf{T}^{n}$. L'image de l'opérateur

$\bar{\partial}_{\mathcal{F}}: \Omega_{\mathcal{F}}^{0,0}\left(\mathbf{T}^{n}\right) \longrightarrow \Omega_{\mathcal{F}}^{0,1}\left(\mathbf{T}^{n}\right)$ s'identifie donc au sous-espace $\mathcal{N}$; en fait la restriction de $\bar{\partial}_{\mathcal{F}}$ à $\mathcal{N}$ est un isomorphisme (algébrique continu) sur $\mathcal{N}$; notons $G_{0}$ son inverse : à $g$ dans $\mathcal{N}$ on associe $f$ unique solution dans $\mathcal{N}$ de l'équation $\bar{\partial}_{\mathcal{F}} f=g \bar{\omega}$. On pose alors $G=G_{0} P$; on vérifie facilement que $G \bar{\partial}_{\mathcal{F}}=I-\mathcal{L}$.

L'inégalité (4) montre que pour tout entier naturel $s$, l'opérateur :

$$
G_{0}: g \in \mathcal{N} \subset W^{1, s+1+2 \tau} \longmapsto G_{0}(g)=f \in C^{\infty}\left(\mathbf{T}^{n}\right) \subset W^{1, s}
$$

vérifie l'inégalité :

$$
\left\|G_{0}(g)\right\|_{1, s} \leq \beta\|g\|_{1, s+1+2 \tau}
$$

Il est donc borné.

Comme on vient de le voir, l'image de l'opérateur $\bar{\partial}_{\mathcal{F}}: C^{\infty}\left(\mathbf{T}^{n}\right) \longrightarrow C^{\infty}\left(\mathbf{T}^{n}\right) \otimes \bar{\omega}$ est l'espace $\mathcal{N} \otimes \bar{\omega}$ qui est de codimension 
1, donc l'espace vectoriel $H_{\mathcal{F}}^{0,1}\left(\mathbf{T}^{n}\right)$ est de dimension 1 engendré par la $(0,1)$-forme $\bar{\omega}$.

ii) $\underline{\mathcal{F} \text { de Liouville }}$

Cela signifie que le couple $(\nu, \kappa)$ est de Liouville. Il existe donc $\delta>0$ tel que pour toute suite strictement croissante $\left(\tau_{k}\right)_{k}$ dans $\mathbf{N}^{*}$, il existe une suite infinie $\left(\mathbf{m}_{k}\right)_{k}$ dans $\mathbf{Z}^{n} \backslash\{\mathbf{0}\}$ vérifiant :

$$
\left|\left\langle\nu, \mathbf{m}_{k}\right\rangle+i\left\langle\kappa, \mathbf{m}_{k}\right\rangle\right| \leq \frac{\delta}{\left|\mathbf{m}_{k}\right|^{\tau_{k}}} .
$$

On définit alors une fonction $g$ à l'aide de ses coefficients de Fourier :

$$
g_{\mathbf{m}}= \begin{cases}\left|\mathbf{m}_{k}\right|^{-\frac{\tau_{k}}{2}} & \text { si } \mathbf{m}=\mathbf{m}_{k} \\ 0 & \text { sinon }\end{cases}
$$

Il est facile de vérifier, à l'aide de l'assertion iii) de la proposition 3.1, que $g$ est de classe $C^{\infty}$. Mais :

$$
\begin{aligned}
\left|f_{\mathbf{m}_{k}}\right| & =\left|\frac{-i \mathbf{m}_{k}}{\pi\left(\left\langle\nu, \mathbf{m}_{k}\right\rangle+i\left\langle\kappa, \mathbf{m}_{k}\right\rangle\right)}\right| \\
& =\frac{\left|\mathbf{m}_{k}\right|^{-\frac{\tau_{k}}{2}}}{\pi\left|\left\langle\nu, \mathbf{m}_{k}\right\rangle+i\left\langle\kappa, \mathbf{m}_{k}\right\rangle\right|} \\
& \geq \frac{1}{\pi \delta}\left|\mathbf{m}_{k}\right|^{\frac{\tau_{k}}{2}} .
\end{aligned}
$$

Les modules des coefficients $f_{\mathbf{m}}$ tendent vers $+\infty$ ! De cette façon on peut fabriquer une famille infinie libre de fonctions $\left(g^{\tau}\right)_{\tau \in \mathbf{N}^{*}}$ de classe $C^{\infty}$ pour lesquelles l'équation (3) n'a pas de solution. Le conoyau de l'opérateur $\bar{\partial}_{\mathcal{F}}: \Omega_{\mathcal{F}}^{0,0}\left(\mathbf{T}^{n}\right) \longrightarrow \Omega_{\mathcal{F}}^{0,1}\left(\mathbf{T}^{n}\right)$ est donc de dimension infinie i.e. l'espace vectoriel $H_{\mathcal{F}}^{0,1}\left(\mathbf{T}^{n}\right)$ est de dimension infinie.

Si $g$ est un polynôme trigonométrique sans terme constant, l'équation (3) a toujours une solution : le problème de la convergence ne se pose pas. Comme l'adhérence du sous-espace engendré algébriquement par ces polynômes est de codimension 1 (c'est l'orthogonal de la fonction constante 1), l'image de l'opérateur $\bar{\partial}_{\mathcal{F}}: \Omega_{\mathcal{F}}^{0,0}\left(\mathbf{T}^{n}\right) \longrightarrow \Omega_{\mathcal{F}}^{0,1}\left(\mathbf{T}^{n}\right)$ n'est pas fermée, donc $H_{\mathcal{F}}^{0,1}\left(\mathbf{T}^{n}\right)$ n'est pas séparé. Ceci montre clairement que $\bar{H}_{\mathcal{F}}^{0,1}\left(\mathbf{T}^{n}\right)=\mathbf{C} \bar{\omega}$.

Remarque et problème 3.4. Tous les résultats du théorème 3.3 restent vrais si on avait considéré le complexe différentiel 
$0 \longrightarrow \Omega_{\mathcal{F}}^{1,0}\left(\mathbf{T}^{n}\right) \stackrel{\bar{\partial}_{\mathcal{F}}}{\longrightarrow} \Omega_{\mathcal{F}}^{1,1}\left(\mathbf{T}^{n}\right) \longrightarrow 0$. En particulier on aurait :

i) si $\mathcal{F}$ est diophantien, l'espace $H_{\mathcal{F}}^{1,1}\left(\mathbf{T}^{n}\right)$ est de dimension 1 engendré par la forme feuilletée $\omega \otimes \bar{\omega}$ qui est de type $(1,1)$;

ii) si $\mathcal{F}$ est de Liouville, l'espace $H_{\mathcal{F}}^{1,1}\left(\mathbf{T}^{n}\right)$ est de dimension infinie non séparé.

Si $\mathcal{F}$ est un feuilletage complexe sur une variété $M$, le fibré vectoriel $T^{10} \mathcal{F} \longrightarrow M$ est $\mathcal{F}$-holomorphe. On peut donc considérer la cohomologie de Dolbeaut feuilletée à valeurs dans $T^{10} \mathcal{F}$ qu'on notera $H_{\mathcal{F}}^{0, *}\left(M, T^{10} \mathcal{F}\right)$. Dans le cas où $M$ est compacte, l'espace vectoriel $H_{\mathcal{F}}^{0,1}\left(M, T^{10} \mathcal{F}\right)$ paramètre les déformations infinitésimales des structures complexes sur $\mathcal{F}$ (les feuilles restent fixes) ( $c f$. [Ek1]) et $H_{\mathcal{F}}^{0,2}\left(M, T^{10} \mathcal{F}\right)$ devrait contenir les obstructions à la réalisation de ces déformations.

Dans le cas du feuilletage $\left(\mathbf{T}^{n}, \mathcal{F}\right)$ qu'on vient d'étudier on a

$H_{\mathcal{F}}^{0,2}\left(\mathbf{T}^{n}, T^{10} \mathcal{F}\right)=0$ (quelle que soit la nature arithmétique de $\mathcal{F}$ ) et $H_{\mathcal{F}}^{0,1}\left(\mathbf{T}^{n}, T^{10} \mathcal{F}\right)=\mathbf{C} \bar{\omega} \otimes Z$ lorsque $\mathcal{F}$ est diophantien.

Problème : On suppose $\mathcal{F}$ diophantien. Montrer que l'espace versel des déformations des structures complexes sur $\mathcal{F}$ est un voisinage de 0 dans $H_{\mathcal{F}}^{0,1}\left(\mathbf{T}^{n}, T^{10} \mathcal{F}\right) \simeq \mathbf{C}$.

\section{Une fibration non localement triviale en courbes ellip- tiques}

Du point de vue différentiable, c'est un feuilletage produit d'une courbe elliptique par un plan mais, du point de vue complexe, il n'est même pas une fibration localement triviale. Toutefois sa cohomologie de Dolbeault feuilletée est exactement celle d'un produit complexe.

\subsection{Construction du feuilletage}

Rappelons que le groupe $\operatorname{Aut}(\mathbf{C})$ des automorphismes de $\mathbf{C}$ est le produit semi-direct $\mathbf{C} \mathbf{C}^{*}$ où le groupe multiplicatif $\mathbf{C}^{*}$ agit par homothéties sur C. Un réseau dans $\mathbf{C}$ est un sous-groupe de la forme $\Gamma^{\prime}=\left\{m_{1} \alpha_{1}+\right.$ $\left.m_{2} \alpha_{2}: m_{1}, m_{2} \in \mathbf{Z}\right\}$ où $\alpha_{1}$ et $\alpha_{2}$ sont des nombres complexes linéairement indépendants sur $\mathbf{R}$. Le quotient $\mathbf{T}^{\prime}=\mathbf{C} / \Gamma^{\prime}$ est une courbe elliptique. Si $\Gamma=\left\{m_{1}+m_{2} \omega: m_{1}, m_{2} \in \mathbf{Z}\right\}$ avec $\omega=\frac{\alpha_{2}}{\alpha_{1}}$, les deux courbes elliptiques $\mathbf{T}^{\prime}=\mathbf{C} / \Gamma^{\prime}$ et $\mathbf{T}=\mathbf{C} / \Gamma$ sont isomorphes, l'isomorphisme $\mathbf{T} \longrightarrow \mathbf{T}^{\prime}$ étant induit par la multiplication $z \in \mathbf{C} \longmapsto \alpha_{1} z \in \mathbf{C}$. 
Le groupe $\Gamma$ étant engendré sur $\mathbf{Z}$ par les vecteurs 1 et $\omega$, on peut toujours choisir $\omega$ dans $\mathbf{H}=\{\omega \in \mathbf{C}: \mathbf{I m} \omega>\mathbf{0}\}$. Dans ce cas $\Gamma=$ $\mathbf{Z} \oplus \omega \mathbf{Z}$ sera noté $\Gamma_{\omega}$ et $\mathbf{T}_{\omega}$ sera la courbe elliptique $\mathbf{T}=\mathbf{C} / \Gamma_{\omega}$. Il est bien connu que les deux courbes elliptiques $\mathbf{T}_{\omega}$ et $\mathbf{T}_{\zeta}$ sont isomorphes si, et seulement si, il existe une matrice $A \in \mathrm{SL}(2, \mathbf{Z})$ telle que $A \omega=\zeta$. Les classes d'isomorphie des courbes elliptiques sont donc paramétrées par la surface modulaire $\mathbf{H} / \mathrm{SL}(2, \mathbf{Z})$.

Le groupe $\operatorname{Aut}(\mathbf{C})$ contient les translations $\tau_{b}: z \longrightarrow z+b$ avec $b \in \mathbf{C}^{*}$. Deux translation $\tau_{b}$ et $\tau_{b^{\prime}}$ sont toujours conjuguées : il suffit de prendre l'homothétie $h: z \in \mathbf{C} \longmapsto a z \in \mathbf{C}$ avec $a=\frac{b}{b^{\prime}}$ et voir que $h^{-1} \circ \tau_{b} \circ h=\tau_{b^{\prime}}$. Par conséquent les sous-groupes $\mathcal{T}_{b}$ et $\mathcal{T}_{b^{\prime}}$ engendrés respectivement par $\tau_{b}$ et $\tau_{b^{\prime}}$ sont conjugués. Les quotients $\mathbf{C} / \mathcal{T}_{b}$ (avec $b$ variant dans $\mathbf{C}$ ) sont donc tous isomorphes à la même surface de Riemann $\mathbf{C}^{*}$.

Soit $p: \mathbf{C} \longrightarrow \mathbf{C}^{*}$ l'application définie par $p(z)=e^{2 i \pi z}$. Alors l'action standard de $\Gamma_{\omega}$ sur $\mathbf{C}$ (i.e. celle qui donne la courbe elliptique $\mathbf{T}_{\omega}$ ) se projette par $p$ en l'action $\Psi$ de $\mathbf{Z}$ sur $\mathbf{C}^{*}$ donnée par $\Psi:(k, z) \in \mathbf{Z} \times$ $\mathbf{C}^{*} \longmapsto e^{2 i k \pi \omega} z \in \mathbf{C}^{*}$. Les courbes elliptiques $\mathbf{T}_{\omega}$ et $C_{\omega}=\mathbf{C}^{*} / \Psi$ sont alors isomorphes.

Soit $\widehat{M}$ la variété complexe $\mathbf{C}^{*} \times \mathbf{H}$. Un point de $\widehat{M}$ sera repéré par ses coordonnées $(z, \omega)$; on utilisera $\left(\omega_{1}, \omega_{2}\right) \in \mathbf{R} \times \mathbf{R}_{+}^{*}$ pour désigner la coordonnée $\omega$. On munit $\widehat{M}$ du feuilletage holomorphe $\widehat{\mathcal{F}}$ dont les feuilles sont les sous-variétés complexes $\left\{\mathbf{C}^{*} \times\{\omega\}\right\}_{\omega \in \mathbf{H}}$. Une fonction $C^{\infty}$ et $\widehat{\mathcal{F}}$ holomorphe est une fonction $f: \widehat{M} \longrightarrow \mathbf{C}$ de classe $C^{\infty}$ par rapport au couple $(z, \omega)$ et holomorphe en $z$. Une telle fonction admet un développement de Laurent en $z$ :

$$
f(z, \omega)=\sum_{p \in \mathbf{Z}} f_{p}(\omega) z^{p}
$$

où les $f_{p}$ sont des fonctions $C^{\infty}$ en $\omega$ et telles que, pour tout compact $K \times C \subset \mathbf{C}^{*} \times \mathbf{H}$ et tout entier naturel $s$ la série de Laurent $\sum_{p \in \mathbf{Z}}\left\|f_{p}\right\|_{\infty}^{s} z^{p}$ converge uniformément par rapport à $z \in K$ où :

$$
\left\|f_{p}\right\|_{K}^{s}=\max _{s_{1}+s_{2} \leq s}\left\{\operatorname{Sup}_{\omega \in C}\left|\frac{\partial^{s} f_{p}}{\partial \omega_{1}^{s_{1}} \partial \omega_{2}^{s_{2}}}(\omega)\right|\right\} .
$$

Cette condition traduit la régularité "être de classe $C^{\infty}$ et $\widehat{\mathcal{F}}$-holomorphe" dans l'espace $\mathcal{H}_{\widehat{\mathcal{F}}}(\widehat{M})$.

Soit $\varphi: \omega=\omega_{1}+i \omega_{2} \longmapsto \omega^{\prime}=\frac{a \omega+b}{c \omega+d}=\omega_{1}^{\prime}+i \omega_{2}^{\prime}$ un biholomorphisme non trivial de $\mathbf{H}$ (où $(a) b c d$ est un élément de $\mathrm{SL}(2, \mathbf{R})$ ). L'application: 
$\left(\begin{array}{ll}a & b \\ c & d\end{array}\right)$ est une action holomorphe, libre et propre de $\mathbf{Z}$ sur $\widehat{M}$. Elle préserve le feuilletage $\widehat{\mathcal{F}}$ (elle préserve même chaque feuille individuellement). L'espace quotient $M=\mathbf{C}^{*} \times \mathbf{H} / \Phi$ est alors muni d'une structure de variété complexe de dimension 2 . On note $\mathcal{F}$ le feuilletage sur $M$ induit par $\widehat{\mathcal{F}}$; il est de dimension 1 et ses feuilles sont toutes des courbes elliptiques $\mathbf{T}_{\omega}$ dont la structure complexe varie en fonction de $\omega \in \mathbf{H}$. Deux feuilles $\mathbf{T}_{\omega}$ et $\mathbf{T}_{\zeta}$ sont isomorphes si, et seulement si, il existe une matrice $B \in \mathrm{SL}(2, \mathbf{Z})$ telle que $\zeta=B \omega$.

La classe d'isomorphie d'une feuille est donc un ensemble dénombrable. Ce feuilletage complexe n'est donc même pas une fibration complexe localement triviale. Mais différentiablement, $\mathcal{F}$ n'est rien d'autre qu'un produit.

Théorème 4.1. L'espace vectoriel de cohomologie $H_{\mathcal{F}}^{0,1}(M)$ est un module libre de rang 1 sur l'anneau $C^{\infty}(\mathbf{H})$ des fonctions $C^{\infty}$ sur $\mathbf{H}$.

Proof. $\quad$ Comme le feuilletage $\widehat{\mathcal{F}}$ est un produit complexe de $\mathbf{H}$ par $\mathbf{C}^{*}$ qui est un ouvert de $\mathbf{C}$ (donc une variété de Stein), pour tout $q \geq 1$, on a :

$$
H_{\widehat{\mathcal{F}}}^{0, q}\left(\mathbf{C}^{*} \times \mathbf{H}\right)=\mathbf{H}^{\mathbf{0}, \mathbf{q}}\left(\mathbf{C}^{*}\right) \otimes \mathbf{C}^{\infty}(\mathbf{H})=\mathbf{0}
$$

D'après $(2)$ on a $H_{\mathcal{F}}^{0,1}(M)=H^{1}\left(\mathbf{Z}, \mathcal{H}_{\widehat{\mathcal{F}}}(\widehat{\mathbf{M}})\right)$ où $\mathcal{H}_{\widehat{\mathcal{F}}}(\widehat{M})$ est vu comme $\mathbf{Z}$-module via l'action :

$$
(p, f) \in \mathcal{H}_{\widehat{\mathcal{F}}}(\widehat{M}) \longmapsto f \circ \gamma^{p} \in \mathcal{H}_{\widehat{\mathcal{F}}}(\widehat{M})
$$

où $\gamma(z, \omega)=\left(e^{-i \varphi(\omega)} z, \omega\right)$. Ce qui nous amène donc à résoudre l'équation cohomologique discrète :

Étant donnée $g \in \mathcal{H}_{\widehat{\mathcal{F}}}(\widehat{M})$ existe-t-il $\left.f \in \mathcal{H}_{\widehat{\mathcal{F}}}(\widehat{M})\right)$ telle que $f-f \circ \gamma=g$ ?

Pour ce faire, nous utiliserons les développements de Laurent respectivement de $f$ et $g$ :

$$
f(z, \omega)=\sum_{p \in \mathbf{Z}} f_{p}(\omega) z^{p} \quad \text { et } \quad g(z, \omega)=\sum_{p \in \mathbf{Z}} g_{p}(\omega) z^{p} .
$$

$\mathrm{Au}$ niveau des coefficients $f_{p}$ et $g_{p}$, l'équation $f-f \circ \gamma=g$ est alors équivalente au système suivant : 


$$
\left(1-e^{-i p \varphi(\omega)}\right) f_{p}(\omega)=g_{p}(\omega) \quad \text { pour } p \in \mathbf{Z} .
$$

Une condition nécessaire d'existence d'une solution est que la fonction $g_{0}$ soit identiquement nulle. Nous allons montrer qu'elle est aussi suffisante. Supposons cette condition remplie. On pose alors :

$$
f_{p}(\omega)= \begin{cases}0 & \text { si } p=0 \\ \frac{g_{p}(\omega)}{1-e^{-i p \varphi(\omega)}} & \text { si } p \neq 0\end{cases}
$$

Reste à montrer que la collection de fonctions $\left(f_{p}\right)_{p \in \mathbf{Z}}$ définit bien une fonction $f \in \mathcal{H}_{\widehat{\mathcal{F}}}(\widehat{M})$. On a $\left|e^{-i p \varphi(\omega)}\right|=e^{p \omega_{2}^{\prime}}=e^{\frac{p \omega_{2}}{|c \omega+d|^{2}}}$.

D'où :

$$
\left|1-e^{-i p \varphi(\omega)}\right| \geq\left|1-e^{\frac{p \omega_{2}}{|c \omega+d|^{2}}}\right|>0
$$

Par suite :

$$
\frac{1}{\mid 1-e^{-i p \varphi(\omega) \mid}} \leq \frac{1}{\left|1-e^{\frac{p \omega_{2}}{|c \omega+d|^{2}}}\right|}
$$

On voit aussi facilement que :

$$
\lim _{p \rightarrow+\infty} \frac{1}{\left|1-e^{\frac{p \omega_{2}}{|c \omega+d|^{2}}}\right|}=0 \quad \text { et } \quad \lim _{p \rightarrow-\infty} \frac{1}{\left|1-e^{\frac{p \omega_{2}}{|c \omega+d|^{2}}}\right|}=1 .
$$

Soit $K \times C$ un compact de $\mathbf{C}^{*} \times \mathbf{H}$. Alors comme la fonction

$\omega \longmapsto \frac{1}{\mid 1-e^{\frac{p \omega_{2}}{|c \omega+d|^{2}}}}$ est en plus continue, il existe $\delta>0$ tel que, pour tout $p \in \mathbf{Z}^{*}$ et tout $\omega \in C$ on ait $\left|f_{p}(\omega)\right| \leq \delta\left|g_{p}(\omega)\right|$. Il en résulte que la série $\sum_{p \in \mathbf{Z}^{*}} f_{p}(\omega) z^{p}$ converge uniformément sur $K \times C$ car la série $\sum_{p \in \mathbf{Z}^{*}} g_{p}(\omega) z^{p}$ y est uniformément convergente.

En utilisant le fait que $g \in \mathcal{H}_{\widehat{\mathcal{F}}}(\widehat{M})$ et en suivant le même type de raisonnement, on montre que, pour tout compact $K \times C \subset \mathbf{C}^{*} \times \mathbf{H}$ et tout entier naturel $s$ la série $\sum_{p \in \mathbf{Z}}\left\|f_{p}\right\|_{\infty}^{s} z^{p}$ converge uniformément par rapport à $z \in K$. Ce qui établit le fait que $f \in \mathcal{H}_{\widehat{\mathcal{F}}}(\widehat{M})$. 
L'image de l'opérateur $f \in \mathcal{H}_{\widehat{\mathcal{F}}}(\widehat{M}) \longmapsto(f-f \circ \gamma) \in \mathcal{H}_{\widehat{\mathcal{F}}}(\widehat{M})$ est donc exactement le noyau de l'application linéaire continue surjective $g \in \mathcal{H}_{\widehat{\mathcal{F}}}(\widehat{M}) \longmapsto g_{0} \in C^{\infty}(\mathbf{H})$. Il en résulte que l'espace :

$$
H_{\mathcal{F}}^{0,1}(M)=H^{1}\left(\mathbf{Z}, \mathcal{H}_{\widehat{\mathcal{F}}}(\widehat{\mathbf{M}})\right)
$$

est un module libre sur $C^{\infty}(\mathbf{H})$ engendré par la fonction constante égale à 1.

Je remercie Aziz El Kacimi de m'avoir soumis ce problème et guidée dans sa résolution.

\section{References}

[1] Brown, K.S. Cohomology of Groups. GTM Vol. 87, Springer-Verlag (1982).

[2] Dederich, K. \& Ohsawa, T. On the parameter dependence of solutions to the $\bar{\partial}$-équation. Math.Ann. 289, pp. 581-588, (1991).

[3] El Kacimi Alaoui, A. Le problème du $\bar{\partial}$ le long des feuilles. Prépublication de l'Université de Valenciennes (Mars 2007).

[4] El Kacimi Alaoui, A. The $\bar{\partial}$ along the leaves and Guichard's Theorem for a simple complex foliation. Prépublication de l'Université de Valenciennes (December 2007).

[5] El Kacimi Alaoui, A. \& Slimène, J. Le $\bar{\partial}_{\mathcal{F}}$ pour certains feuilletages complexes. Prépublication Université de Valenciennes, Mars, (2008).

[6] Gigante, G. \& Tomassini, G. Foliations with complex leaves. Diff. Geo. and its Applications 5, pp. 33-49, (1995).

[7] Grothendieck, A. Sur quelques points d'algèbre homologique. Tôhoku Math. J. Vol. 9, pp. 119-221, (1957).

[8] Schmidt, W. M. Diophantine approximation. Lecture Notes in Math. $785,(1980)$. 


\section{Jihène Slimène}

Département de Mathématiques

Faculté des Sciences de Monastir

Boulevard de l'Environnement

5919 Monastir - Tunisie

e-mail : jihene.slimene@yahoo.fr 